\title{
AMENDMENTS
}

\section{Publisher Correction: Laser-plasma interactions: To degeneracy and back}

\section{Adam J. Burgasser (D)}

Correction to: Nature Physics https://doi.org/10.1038/s41567-020-0860-6, published online 9 March 2020.

In the version of this News \& Views article originally published, in ref. 3, the URL was missing an 's' and should have read 'https://doi. org/10.1038/s41567-020-0790-3', also '(2019)' should have been '(2020)'. The online versions of the article have been amended.

Published online: 14 April 2020

https://doi.org/10.1038/s41567-020-0897-6

() Springer Nature Limited 2020

\section{Author Correction: Unveiling the predictive power of static structure in glassy systems \\ V. Bapst (D), T. Keck, A. Grabska-Barwińska, C. Donner, E. D. Cubuk, S. S. Schoenholz, A. Obika, A. W. R. Nelson, T. Back, \\ D. Hassabis and P. Kohli}

Correction to: Nature Physics https://doi.org/10.1038/s41567-020-0842-8, published online 6 April 2020.

In the version of this Article originally published, the URL given in the data and code availability statements was incorrect as https:// github.com/deepmind/deepmind-research/glassy-dynamics, and should have been https://github.com/deepmind/deepmind-research/ tree/master/glassy_dynamics. The online versions of the article have been amended.

Published online: 16 April 2020

https://doi.org/10.1038/s41567-020-0901-1

(c) The Author(s), under exclusive licence to Springer Nature Limited 2020

\section{Author Correction: Higher-order topology in bismuth}

Frank Schindler, Zhijun Wang (D), Maia G. Vergniory, Ashley M. Cook, Anil Murani, Shamashis Sengupta, Alik Yu. Kasumov, Richard Deblock, Sangjun Jeon, Ilya Drozdov, Hélène Bouchiat, Sophie Guéron, Ali Yazdani (D), B. Andrei Bernevig and Titus Neupert (D)

Correction to: Nature Physics https://doi.org/10.1038/s41567-018-0224-7, published online 30 July 2018.

In the version of this Article originally published, in the Acknowledgements, for author B.A.B. the support from the Department of Energy should have been listed separately from the other acknowledgements. The sentence 'B.A.B. acknowledges support for the analytic work from the Department of Energy (de-sc0016239), the Simons Investigator Award, the Packard Foundation, and the Schmidt Fund for Innovative Research.' should have been 'B.A.B. acknowledges support for the analytic work from the Department of Energy (de-sc0016239). B.A.B. also acknowledges support from a Simons Investigator Award, the Packard Foundation, and the Schmidt Fund for Innovative Research.' The online versions of the article have been amended.

Published online: 15 April 2020

https://doi.org/10.1038/s41567-020-0902-0

(c) The Author(s), under exclusive licence to Springer Nature Limited 2020 